\title{
Circulatory support with Impella CP device during high-risk percutaneous coronary interventions: initial experience in Poland
}

\author{
Dariusz Dudek ${ }^{1}$, Tomasz Rakowski², Adam Sukiennik ${ }^{3}$, Michat Hawranek', Artur Dziewierz², Jacek Kubica ${ }^{3}$, \\ Piotr Suwalski ${ }^{5,6}$, Robert Gil7, Wojciech Wojakowski ${ }^{8}$, Andrzej Ochała ${ }^{8}$, Wiesław Mazurek', \\ Krzysztof Żmudka ${ }^{1}$, Mariusz Gąsior ${ }^{4}$
}

\begin{abstract}
${ }^{1}$ Department of Interventional Cardiology, Jagiellonian University Medical College, Institute of Cardiology, Krakow, Poland ${ }^{2}$ Second Department of Cardiology, Jagiellonian University Medical College, Institute of Cardiology, Krakow, Poland

${ }^{3}$ Department of Cardiology and Internal Medicine, Collegium Medicum in Bydgoszcz, Nicolaus Copernicus University in Torun, Poland ${ }^{4}$ Third Department of Cardiology, Silesian Centre for Heart Disease, Medical University of Silesia, Zabrze, Poland

${ }^{5}$ Department of Cardiac Surgery, Central Clinical Hospital of the Ministry of Interior, Warsaw, Poland

${ }^{6}$ Faculty of Health Science and Physical Education, Pulaski University of Technology and Humanities, Radom, Poland

${ }^{7}$ Department of Invasive Cardiology, Central Clinical Hospital of the Ministry of Interior, Warsaw, Poland

${ }^{8}$ Third Department of Cardiology, Medical University of Silesia, Katowice, Poland

${ }^{9}$ Department of Interventional Cardiology, L. Rydygier Hospital, Torun, Poland
\end{abstract}

\section{Introduction}

Coronary revascularization is an important part of the treatment of patients with coronary artery disease. However, a significant proportion of patients are characterized by high-risk features. Many of these patients are referred for high-risk percutaneous coronary interventions ( $\mathrm{PCls}$ ) due to the extremely high risk of surgery. To support such procedures and to facilitate the care of high-risk patients, percutaneous left ventricular assist devices (pLVAD) were developed. Due to confounding data and downgraded guidelines for use of the intra-aortic balloon pump (IABP), especially in cardiogenic shock caused by myocardial infarction (MI), there is currently growing interest in $\operatorname{pLVAD}[1,2]$. The use of pLVAD during high-risk $\mathrm{PCI}$ in Europe varies from country to country mainly due to different reimbursement policies.

\section{Aim}

Due to growing interest in pLVAD support during high-risk PCls in Poland and Europe, we aim to present our initial experience with the Impella CP system during high-risk PCls.

\section{Material and methods}

The study is a prospective registry of all patients treated with the Impella CP (ABIOMED Inc., Danvers, MA, USA) in Poland. Data are collected based on a dedicated questionnaire. The registry is currently conducted in six high-volume $\mathrm{PCl}$ centers. All procedures are performed with highly experienced operators and are fully supported by company (distributor in Poland) staff either on site for elective procedures or with phone support for emergency usage. Also two types of training courses are provided for physicians, nurses and technicians: one for staff performing the procedure with the Impella and the second for staff taking care of patients after the procedure. This is important for the first cases when this new technology is implemented.

The results are presented as the number of patients or mean \pm standard deviation where applicable.

\section{Results}

A total of 10 patients were treated with Impella CP support from April 2013 to August 2015 (four in 2013/14 and six in 2015). In all cases except one, the Impella CP was used during high-risk elective $\mathrm{PCl}$, and the present

\section{Corresponding author:}

Dariusz Dudek MD, PhD, Department of Interventional Cardiology, Jagiellonian University Medical College, Institute of Cardiology, 17 Kopernika St, 31-501 Krakow, Poland, phone: +48 12424 71 81, fax: +48 1242471 84, e-mail: mcdudek@cyfronet.pl

Received: 1.03.2016, accepted: 14.06.2016. 
analysis is focused on those nine patients. In this one excluded case, the Impella CP was used due to an acute MI complicated with cardiogenic shock. Baseline characteristics of enrolled patients are summarized in Table I. All patients were men with a mean age of $73 \pm 11$ years and mean left ventricular ejection fraction of $32 \pm 8 \%$. The baseline risk profile of enrolled patients was rather high, with previous revascularization, previous $\mathrm{MI}$ and chronic kidney disease in half of the patients. In the majority of cases the left main coronary artery was the target vessel for $\mathrm{PCl}$ (Table I). All patients received drug-eluting stents during $\mathrm{PCl}$. In all cases the device was implanted under the supervision of the company representative on site. In all cases the femoral access with a 14 F Oscor introducer and Abiomed 0.018 guidewire was used. In two cases the Impella CP was implanted with surgeon support and in seven with the wire insertion method. The Automated Impella Controller was used in all patients with Autoflow and P-level configuration in 5 and 4 patients respectively. In all cases support with the Impella CP was continued until the end of the procedure. Surgical closure was used in four patients and an arterial closure device in 5 patients (four with Perclose ProGlide (Abbott Vascular, CA, USA), one with AngioSeal (St. Jude Medical, MN, USA)). Thirty-day outcomes of patients treated for high-risk elective $\mathrm{PCl}$ were good, with no death during follow-up. Only in 1 patient was a small hematoma at the site of device insertion noted.

\section{Discussion}

In our initial series we found the use of the Impella CP during high-risk $\mathrm{PCl}$ to be feasible and safe.

Patients with extensive coronary artery disease, depressed left ventricular ejection fraction, hemodynamic instability and multiple comorbidities are at high risk for coronary revascularization procedures. During recent years due to population aging in Europe there is a growing number of such high-risk elderly patients who are candidates for revascularization. Coronary artery bypass grafting with full revascularization seems to be a preferred option, but often due to high surgical risk such patients are referred for percutaneous revascularization $[3,4]$. In such a case the benefit of percutaneous revascularization is expected; however, the risk of periprocedural events is very high. Therefore there is a strong clinical need of periprocedural support. Formerly, IABP was used as a $\mathrm{PCl}$ support mainly for patients with $\mathrm{MI}$ and/ or cardiogenic shock. It was used less often during elective high-risk procedures. However, according to current data the value of IABP is questionable [1, 2]. To overcome the limitations of IABP and to reduce the risk of PCls in such patients, the strategy of procedural support with pLVAD technology was proposed. The Impella technology is an axial flow, rotary pump built on a $9 \mathrm{Fr}$ catheter. For $\mathrm{PCl}$ support, the device is deployed via femoral access

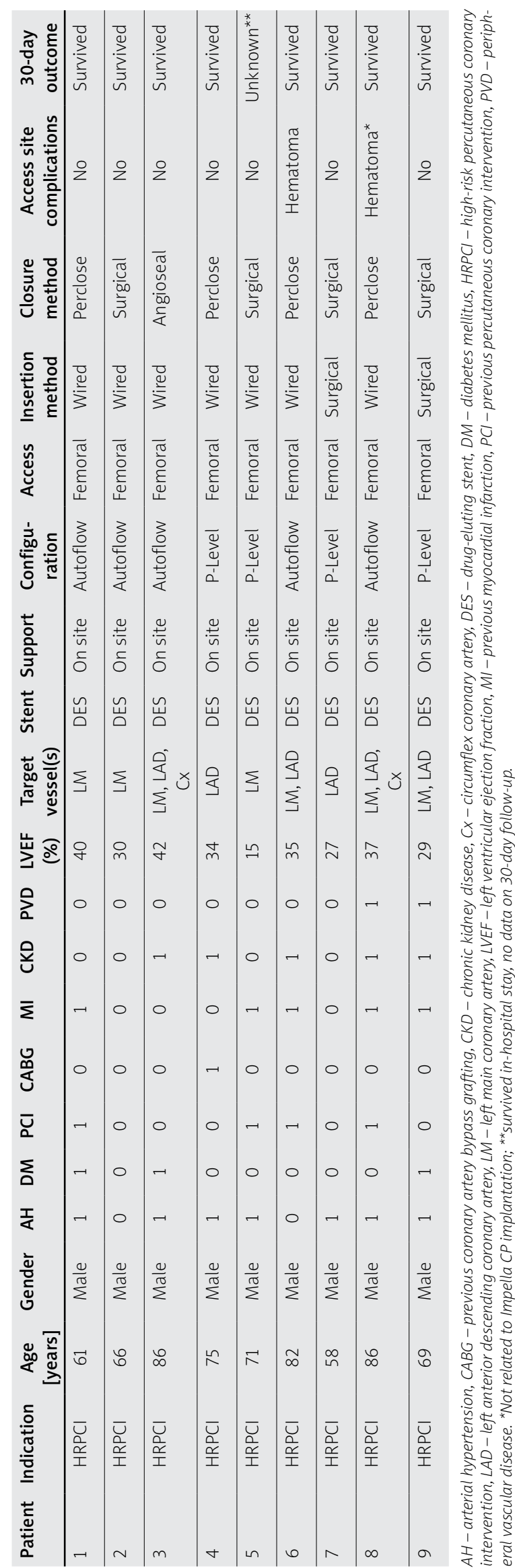


in a retrograde way across the aortic valve. In our series of patients the Impella CP with higher blood flow was used. The Impella CP theoretically provides nonpulsatile forward blood flow of up to $4.0 \mathrm{l} / \mathrm{min}$ (max speed 46000 rpm) which is independent of cardiac rhythm. However, there is native heart pulsatile flow creating pulsatility of blood flow through the device. That is why the motor current maximum is reached during systole and is associated with higher flows and higher pump speeds. So pump flow is the highest during systole and lowest during diastole [5]. In our series of cases in five patients the "Autoflow" and in four the "P-level" pump configuration was used. Autoflow is a fully automated program in which the device provides maximal support for the particular patient, and there is no need for operator intervention. This may be a reasonable option for initial cases. P-level configuration is a manual mode in which a support level from P0 to P9 may be selected, but some experience is necessary to use such a protocol. This mode is also useful for device removal, especially for cardiogenic shock patients when support should be gradually reduced (like in IABP) before termination. Importantly, the device is designed for a fully percutaneous femoral approach with no need of surgical preparation. However, in the presented results surgical vascular access was used in 2 cases in one study center, but it was according to the local policy for new device introduction rather than a systemic approach. In 4 cases surgical vessel closure was performed for the same reason. For high-risk PCl support, vascular access and vascular closure after Impella usage may be used fully percutaneously by an operator who is experienced in this technology. For initial cases, surgical back-up may be a reasonable option. In Poland, the first procedure of percutaneous Impella support (Impella 2.5 device) was performed in the Institute of Cardiology in Krakow (K. Żmudka, T. Pawelec) in 2007. In the PROTECT II study, Impella technology was shown to be associated with improved clinical outcome at 90 days as compared to IABP in patients undergoing high-risk $\mathrm{PCI}$ [6]. In a sub-analysis of PROTECT II the benefit of Impella (compared to IABP) was observed in patients with three-vessel coronary artery disease and impaired left ventricular function [7]. The PROTECT II study was based on the Impella 2.5 device. In the Europella Registry on high-risk patients with a logistic EuroSCORE of about 15\%, the 30-day death rate was $5.5 \%$, the vascular complication rate was $4 \%$, and device malfunction was not observed, showing Impella 2.5 periprocedural support to be safe and feasible [8]. Similarly, in the USpella registry the overall angiographic success was $99 \%$, the survival rate was $96 \%$ at 30 days and $88 \%$ at 12 months, and in $3.4 \%$ of patients transfusion was required due to access site bleeding [9]. Despite the higher risk of the patients included in the USpella registry as compared to those enrolled in the randomized trial (PROTECT II), clinical outcomes of registry "reallife" patients appeared to be favorable [10]. Operator's experience seems to be important for patient outcomes when using Impella support during high-risk $\mathrm{PCI}$. In the prespecified subgroup analysis of the PROTECT II study in which the outcomes were evaluated after excluding the first Impella and IABP patients at each site, a trend toward higher rates of major adverse cardiovascular events at 30 days was observed for the subgroup of the first versus the remaining Impella 2.5 patients. Importantly, after exclusion of the first patient in each group, the major adverse cardiovascular event rate for the Impella 2.5 was significantly lower compared to IABP at 90 days (38\% vs. $50 \% ; p=0.029$ ) [11]. This may suggest the presence of a learning curve associated with the use of the Impella 2.5. So it may be better to perform the first cases with the supervision of an experienced proctor. This fact also raises a question about the hub-spoke concept for $\mathrm{PCl}$ procedures with Impella support. It may be reasonable to establish reference centers to increase the team experience and improve the results by cumulating the usage of the device in a limited number of centers. It should be underlined that such a scenario is possible in planned $\mathrm{PCl}$ procedures but probably not in patients with cardiogenic shock, who are also a target population for pLVAD support [12].

The presented strategy describes the so-called "protected PCI". In such an approach, complex PCI procedures in high-risk patients may be performed with a full range of devices and techniques due to the stable hemodynamic status driven by the pLVAD support. This may improve patients' outcome. However, the penetration of PLVAD is dependent on the reimbursement policy. In Poland this procedure is currently not reimbursed, and it is fully covered by hospital funds. Impella usage during high-risk PCI is reimbursed in many European countries and the USA.

It is worth mentioning that besides LV support it is also possible to provide right ventricle support with the Impella RP system. It may be used in patients with cardiogenic shock due to RV failure and may be helpful either in isolation or in combination with the Impella CP for patients with shock [13].

Based on previous studies as well as on the presented experience, the Impella CP seems to be feasible and safe as circulatory support during high-risk $\mathrm{PCI}$ procedures.

\section{Conflict of interest}

The authors declare no conflict of interest.

\section{References}

1. Patel MR, Smalling RW, Thiele $\mathrm{H}$, et al. Intraaortic balloon counterpulsation and infarct size in patients with acute anterior myocardial infarction without shock: the CRISP AMI randomized trial. JAMA 2011; 306: 1329-37

2. Thiele H, Zeymer U, Neumann FJ, et al. Intraaortic balloon support for myocardial infarction with cardiogenic shock. N Engl J Med 2012; 367: 1287-96. 
3. Cohen MG, Filby SJ, Roe MT, et al. The paradoxical use of cardiac catheterization in patients with non-ST-elevation acute coronary syndromes: lessons from the Can Rapid Stratification of Unstable Angina Patients Suppress Adverse Outcomes With Early Implementation of the ACC/AHA Guidelines (CRUSADE) Quality Improvement Initiative. Am Heart J 2009; 158: 263-70.

4. Smith PK, Califf RM, Tuttle RH, et al. Selection of surgical or percutaneous coronary intervention provides differential longevity benefit. Ann Thorac Surg 2006; 82: 1420-8.

5. Al-Rashid F, Nix C, Erbel R, et al. Tools \& Techniques - clinical: percutaneous catheter-based left ventricular support using the Impella CP. Eurolntervention 2015; 10: 1247-9.

6. Dangas GD, Kini AS, Sharma SK, et al. Impact of hemodynamic support with Impella 2.5 versus intra-aortic balloon pump on prognostically important clinical outcomes in patients undergoing high-risk percutaneous coronary intervention (from the PROTECT II randomized trial). Am J Cardiol 2014; 113: 222-8.

7. Kovacic JC, Kini A, Banerjee S, et al. Patients with 3-vessel coronary artery disease and impaired ventricular function undergoing $\mathrm{PCl}$ with Impella 2.5 hemodynamic support have improved 90-day outcomes compared to intra-aortic balloon pump: a substudy of the PROTECT II trial. J Interv Cardiol 2015; 28: 32-40.

8. Sjauw KD, Konorza T, Erbel R, et al. Supported high-risk percutaneous coronary intervention with the Impella 2.5 device the Europella registry. J Am Coll Cardiol 2009; 54: 2430-4.

9. Maini B, Naidu SS, Mulukutla S, et al. Real-world use of the Impella 2.5 circulatory support system in complex high-risk percutaneous coronary intervention: the USpella Registry. Catheter Cardiovasc Interv 2012; 80: 717-25.

10. Cohen MG, Matthews R, Maini B, et al. Percutaneous left ventricular assist device for high-risk percutaneous coronary interventions: real-world versus clinical trial experience. Am Heart 2015; 170: 872-9.

11. Henriques JP, Ouweneel DM, Naidu SS, et al. Evaluating the learning curve in the prospective randomized clinical trial of hemodynamic support with Impella 2.5 versus intra-aortic balloon pump in patients undergoing high-risk percutaneous coronary intervention: a prespecified subanalysis of the PROTECT II study. Am Heart J 2014; 167: 472-9.

12. Pyka L, Pres D, Przybylski R, et al. Mechanical circulatory support in cardiogenic shock - what every interventional cardiologist should know. Postep Kardiol Inter 2014; 10: 195-200.

13. Anderson MB, Goldstein J, Milano C, et al. Benefits of a novel percutaneous ventricular assist device for right heart failure: the prospective RECOVER RIGHT study of the Impella RP device. J Heart Lung Transplant 2015; 34: 1549-60. 